\title{
Breast Cancer: Detection Markers, Prognosis, and Prevention
}

\author{
Murtaza Mustafa ${ }^{1}$, A.Nornazirah ${ }^{2}$, FA.Salih ${ }^{3}$, EL.Illzam ${ }^{4}$, \\ M.Suleiman ${ }^{5}$, AM.Sharifa $^{6}$ \\ ${ }^{1,2,3}$ Faculty of Medicine and Health Sciences, UniversityMalaysia,Sabah,Kota Kinabalu,Sabah,Malaysia. \\ ${ }^{4}$ Clinic Family Planning Association,Kota Kinabalu,Sabah,Malaysia. \\ ${ }^{5}$ Public Health Division, Ministry of Health,Sabah,KotaKinabalu,Sbah,Malaysia. \\ ${ }^{6}$ Quality Unit Hospital Queen Elizabeth,KotaKinabalu,Sabah,Malaysia
}

\begin{abstract}
Breast cancer is the common invasive cancer with high mortality worldwide. High incidence of breast cancer in South and central America,Southren,Northren,WestrenEurope,Ocenia and North America.Lowest breast cancer incidence in Africa and Asia.Risk factors includes: female sex old age, lifestyle, oralcontraceptive, hormone replacement therapy,mutations in the breast cancer susceptibility genes BRCAlorBRCA2.alcohol intake, hereditaryfactors, and exposure to chemicals.Breast cancer occurs because of an interaction between external factor and genetically susceptible host.Frequent symptoms of breast cancer is typically a lump and lumps found in the lymph node in the armpits. Diagnosis by physical examination of the breast and mammography. Further tests include histopathologicalexamination, breast cells grading by TNM systeme.g.,Zero stage a precancerous or marker condition, stage 1- 3 within the breast and regional nodes, and stage four is metastatic stage. Management of breast cancer depends on the stage of the cancer and age of the patient.Usually treated with surgery, chemotherapy or radiation therapy or both. A multidisciplinary approach is preferable. Metastatic cancer has less favorable prognosis. Prognosis is usually the probability of progression-free survival(PFS) or disease free survival(DFS).Prevention include change in life style, maintaining healthy weight, less alcohol consumption, and intake of marine omega-3 and soy-based foods Prophylactic mastectomy(removal of both breasts) helps in people with BRCA1 and BRCA2 mutations. Early detection of breast cancer has better prognosis.
\end{abstract}

Keywords: Breast cancer, Risk factors, Diagnosis, Prognosis

\section{Introduction}

Breast cancer is cancer that develops from breast tissues[1].Early signs of breast cancer may include a lump in the breast, a change in breast shape, dimpling of the skin, fluid coming from the nipple, or a red scaly patch of skin[2].Worldwide breast cancer is the most common invasivecancer in women[3].Breast cancer comprises $22.9 \%$ of invasive cancers in women[4].In 2012, it comprised $25.2 \%$ of cancers diagnosed in women making it the most common female cancer[5].In 2008, breast cancer caused 458,503 deaths worldwide, $13.7 \%$ of cancer deaths in women and 6.\% of all cancer deaths for men and women together[4].In 2012,it resulted in 1.68 million cases and 522,000 deaths[6].Incidence of breast cancer varies greatly around the world: it is lowest in the less-developed countries and greatest in the more- developed countries. In the twelve world regions, theannual age-standardized incidence rates per 10,000 women are :in Eastern Asia,18,South Central Asia,22,sub-Saharan Africa,22,South-Eastern Asia,26,North Africa and Western Asia,28,South and Central America,42,Eastren Europe,49,Southern Europe,56;Nothern Europe,73;Ocenia,74,Wstern Europe,78,North America 90[7].In 2006, Malaysia reported 3525 cases of breast cancer, and 16.5\% of all cancer registered with peak age of 50 years to 59 years age group[8].Breast cancer is strongly related to age with only $5 \%$ of all breast cancers occurring in women under 40 years old[9].Risk factors for developing breast cancer include: female sex, obesity, lack of physical exercise, drinkingalcohol, hormone replacement therapy, ionizingradiationold age and family history[2,].Diagnosis of breast cancer is confirmed by biopsy of the concerning lump, with further tests to determine if the cancer has spread beyond the breast[2]. Outcomes for breast cancer vary depending on the cancer type, extent of disease, and person's age[10].Survival rates in the developed world are high, with between $80 \%$ and $90 \%$ of those in England and the United States alive for at least 5 years[11,12].Management mainly by surgery, medication, and radiation.Preventionby life style changes, maintaining healthy weight and less consumption of alcohol[13].The paper reviews the risk factors, diagnosis, and current management of breast cancer.

\section{Historical Perspectives}

The oldest evidence of breast cancer was discovered in Egypt in 2015 and dates back to Sixth Dynasty [14].The study of a women's remains from necropolis of Qubbet el-Hawa showed the typical destructive 
damage due to metastatic spread[14].The Edwin Smith Payrus describes 8 cases of tumors or ulcers of the breast that were treated by cauterization.The writing says about the disease, "There is no treatment"[15].Ancient medicine, from the time of the Greeks through the $17^{\text {th }}$ century, was based on humoralism,and thus believed that breast cancer was generally caused by imbalances in the fundamental fluids that controlled the body, especially an excess of black bile[16].Alternatively. Patients often saw it as divine punishment [17].

In the $18^{\text {th }}$ century, a wide variety of medical explanations were proposed, including a lack of sexual activity, too much sexual activity, physical injuries to the breast, curdled breast milk, and various forms of lymphatic blockage, either internal or due to restrictive clothing[15].in the $19^{\text {th }}$ century, the Scottish surgeon John Rodman said that fear of cancer caused cancer, and this anxiety, learned by example from mother, accounted for breast cancer's tendency to run in the families[18].Although breast cancer was known in ancient times, it was uncommon until $19^{\text {th }}$ century, when improvements in sanitation and control of deadly infectious diseases resulted in dramatic increase in lifespan. Previously, most woman had died too young to have developed breast cancer [18].Additionally, early frequent childbearing and breastfeeding probably reduced the rate of breast cancer development in those woman who did survive to middle age [18].

Mastectomy for breast cancer was performed at least as early as AD 548, when it was proposed by the court physician Aetios of Amida of Theodora[19].It was not until doctors achieved greater understanding of the circulatory system in the $17^{\text {th }}$ century that they could link breast cancer's spread to lymph nodes in the armpit. The French surgeon Jean Petit(1674-1750) and later Scottish surgeon Benjamin Bell (1749-1806) were the first to remove the lymph nodes, breast tissue, and underlying breast muscle[20].Their successful work was carried on by William Stewart Halsted who started performing radical mastectomies in 1882.This often led to long term pain and disability, but was seen as necessary in order toprevent the cancer recurring[21].Before the advent of the Halsted radical mastectomy,20 year survival rates were only $10 \%$,Halsted surgery raised that rate to 50\%[22].Extending Halsted work, Jerome Urban promoted super radicalmastectomies, taking even more tissue, until 1963, when ten -year survival rates proved equal to the less damaging radical mastectomy[21].

Breast cancer staging systems were developed in the 1920s and 1930s[21].During the 1970s,a new understanding of metastasis led to perceiving cancer as a systemic illness as well as a localized one, and more sparing procedures were developed that proved equally effective. Modern chemotherapy developed after World War 11[23].In 1926,Janet Lane-Claypon published a comprehensive study of 500 breast cancer cases and 500 control patients of the same background and lifestyle for the British Ministry of Health[24].In the 1980s and 1990s, thousands of women who had successfully completed standard treatment then demanded and received high-dose bone marrowtransplants, thinking this would lead to better long-term survival. However, it proved completely ineffective, and 15-20\% of women died because of brutal treatment [25].In 1995 reports from the Nurses 'Health Study and the 2002 conclusions of the Women's Health Initiative trial conclusively proved that hormone replacement therapy significantly increased the incidence of breast cancer[25].

\section{Contributory Factors}

Contributory or risk factors to breast cancer can be divided into two categories:[26].

a) Modifiable risk factors-things that people can change themselves, such as consumption of alcohol beverages.

b) Fixed risk factors-things that cannot be changed, such as age and biological sex.

The primary risk factors for breast cancer are female sex and older age[27].0ther potential risk factors include: genetics, lack of child bearing or lack of breastfeeding[28,29], higher levels of certain hormones, and certain dietary patterns, and obesity[30,31].Recent studies have indicated that exposure to high pollution is a risk factor for the development of breast cancer[32].

\section{Lifestyle factors}

Smoking tobacco appears to increase the risk of breast cancer, with greater the amount smoked and earlier in life that smoking begin, the higher the risk[33].In those who are long term smokers, the risk is increased $35 \%$ to $50 \%$ [33].A lack of physical activity has been linked to $10 \%$ of cases[34].Sitting regularly for prolonged periods is associated with high mortality from breast cancer. The risk is not negated by regular exercise although it is lowered [35].

\section{Oral contraceptives}

There is an association between use of hormonal birth control and the development of premenopausal breast cancer[26,], but whether oral contraceptives use may actually cause premenopausal breast cancer is matter of debate[36].If there is indeed a link, the absolute effect is small[36].Additionally it is not clear if association exists with newer hormonal birth controls[37].In those with mutations in the breast cancer susceptibility genes BRCA1 or BRCA2,or who have a family history of breast cancer, use of modern oral contraceptives does not appear to affect the risk of breast cancer[38]. 


\section{Breast Feeding}

The association between breast feeding and breast cancer has not been determined, some studies have found support for an association while others have not[39].In the 1980s, the abortion-breast cancer hypothesis posited that induced abortion increased the risk of development breast cancer[40]. This subject was the subject of extensive scientificinquiry, which concluded that neither miscarriage nor abortions are associated with a heightened risk for breast cancer[41].

\section{Dietary factors}

A number of dietary factors have been linked to the risk for breast cancer. Dietary factors which may increase risk include a high fat diet, high alcohol intake, and obesity related high cholesterol levels [4244].Dietary iodine deficiency may also play a role[45].Evidence for fiber in unclear. A 2015 review found that studies trying to link fiber intake with breast cancer produced mixed results [46].In 2016 a tentative association between low fiber intake during adolescence and breast cancer was observed [47].

\section{Role of Genetics}

Some genetic susceptibility may play a minor role in most cases [48].Overallhowever,genetics is believed to be the primary cause of 5- 10\% of all cases[49]. Women whose mother was diagnosed before 50 have an increased risk of 1.7 and those whose mother was diagnosed at age 50 or after has an increased risk of 1.4[50].In those with zero,one or two affected relatives, the risk of breast cancer before the age of 80 is $7.8 \%, 13.3 \%$ and $21.1 \%$ with a subsequent mortality from the disease of $2.3 \%, 4.2 \%$ and $7.6 \%$ respectively[51].In those with a first degree relative with disease the risk of breast cancer between the age of 40 and 50 is double that of the general population.[52].

In less than $5 \%$ of cases, genetics plays a more significant role by causing a hereditary breast-ovarian cancer syndrome[48].This includes those who carry the BRCA1 and BRCA2 gene mutation[48].These mutations account for up to $90 \%$ of the total genetic influence with a risk of breast cancer of $60-80 \%$ in those affected[49].Other significant mutations include p53(Li-Fraumeni syndrome),PTEN(Cowden syndrome) and STK11(Peutz-Jeghers syndrome),CHEK2,ATM,BRIP, and PALB2[49].In 2012, researchers said that there are four genetically distinct types of the breast cancer and that in each type, hallmark genetic changes lead to many cancers[53].

\section{Miscellaneous factors and Medical issues}

Other risk factors include radiations, and shift work[54,55].A number of chemicals have also been linked including polychlorinated biphenyls,(PCB),polycyclic aromatic hydrocarbons, organic solvents[56].Although the radiation from mammography is a low dose, it is estimated that yearly screening from 40 to 80 years of age will, cause approximately 225 cases of fatal breast cancer per million women screened $[57]$.

Breast changes like atypical ductal hyperplasia, and lobular carcinoma in situ found in benign breast conditions such as fibrocystic breast, are correlated with an increased breast cancer risk[58,59].Diabetes mellitus might also increase the risk of breast cancer[60].

\section{Pathophysiology}

Breast cancer, like other cancers, occurs because of an interaction between an environmental (external) factor and a genetically susceptible host. Normal cells divide as many times as needed and stop. They attach to other cells and stay in place in tissues. Cells become cancerous when they lose their ability to stop dividing, to attach to other cells, to stay where they belong, and to die at the proper time.

Normal cells will commit cell suicide (programmed cell death) when they are no longer needed. Until then they are protected from cell suicide by several protein clusters and pathways. One of the protective pathways is the P13K/AKT pathway is the RAS/MEK/ERK pathway. Sometimes the genes along these protective pathways are mutated in way that turns them permanently "on", rendering the cell incapable of committing suicide when no longer needed. This is one of the steps that cause cancers in combination with other mutations. Normally, the PTEN protein turns off the P13K/AKT pathway when cell is ready for programmed cell death. In some breast cancers, the gene for the PTEN protein is mutated, so the P13K/AKT pathway is stuck in the: on" position, and the cancer cell does not commit suicide [61].Mutations that can lead to breast cancer have been experimentally linked to estrogen exposure[62].Abnormal growth factor signaling in the interaction between stromal cells and epithelial cells can facilitate malignant cell growth[63].In breast adipose tissue, overexpression of leptin leads to increased cell proliferation and cancer[64].

In the United States, 10 to 20 percent pf people with breast cancer and people with ovarian cancer have first or second degree relative with one of these diseases. The familial tendency to develop these cancers is 
called hereditary breast-ovarian cancer syndrome. The best known of these BRCAmutations, confer a life time risk of breast cancer of 60 and 85 percent and a life time risk of ovarian cancer of between 15 and 40 percent. Some mutations associated with cancer, such as $p 53, B R C A 1$ and $B R C A 2$,occur in mechanisms to correct errors in DNA. These mutations are either inherited or acquired after birth. Presumably, they allow further mutations, which allow uncontrolled division, lack of attachment, and metastasis to distant organs [54].

However, there is strong evidence of residual risk variation that goes well beyond hereditary BRCA gene mutations between carrierfamilies. This is caused by unobserved risk factors [65].This implicates environmental and other causes as triggers for breast cancers. The inherited mutation in BRCA1 or BRCA2 genes can interfere with repair of DNA cross links and DNA double strand breaks(known functions of encoded protein) [66]. These carcinogens cause DNA damage such as DNA cross links and double strand breaks that often require repairs by pathways containing BRCA1 and BRCA2[67].However, mutations in BRCA genes accounts for only 2 to 3 percent of all breast cancers[68].Levin et al say that cancer may not be inevitable for all carriers of BRCA1 and BRCA2 mutations. About half of hereditary breast-ovarian cancer syndrome involve unknown genes.[69].GATA-3 directly controls the expression of estrogen receptor(ER) and other genes associated with epithelial differentiation, and the loss of GATA-3 leads to loss of differentiation and poor prognosis due to cancer cell invasion and metastasis[70].

\section{Clinical Manifestations}

The first noticeable symptom of breast cancer is typically a lump that feels different from the rest of the breast tissue. More than $80 \%$ of breast cancer are discovered when the woman feels a lump [71].The earliest breast cancers are detected by a mammogram. Lumps found in lymph node located in the armpits can also indicate breast cancer[72,71].Indications of breast cancer other than a lump may include thickening different from other breast tissue, one breast becoming larger or lower, a nipple changing position or shape or becoming inverted, skin puckering or dimpling, a rash on or around nipple, discharge from nipple/constant pain in part of breast or armpit, and swelling beneath the armpit or around the collarbone[73].Pain("mastodynia")is an unreliable tool in determining the presence or absence breast cancer, but may be indicative of other breast health issues[71,72,74].

Another reported symptom complex of breast cancer is Paget's disease of the breast. This syndrome presents as skin changes resembling eczema, such as redness, discoloration, or mild flaking of the nipple. As Paget's disease advances, symptoms may include tingling, itching,,increased sensitivity, burning,andpain.There may also be discharge from the nipple. Approximately half of women with diagnosed with Paget's disease of the breast also have a lump in the breast [75].

Occasionally, breast cancer presents as metastatic disease-that is, cancer that has spread beyond the original organ. The symptoms of caused by metastatic breast cancer will depend on the location of metastasis. Common sites od metastasis include bone, liver, lung, and brain[76].Unexplained weight loss can occasionally signal breast cancer, as can symptoms of fevers or chills. Bone or joint pains can sometimes be manifestations of metastatic breast cancer, such as can jaundice or neurological symptoms. These symptoms are called nonspecific, meaning they could be manifestations of many other illnesses [77].

Fewer than $20 \%$ of lumps, forexample, arecancerous, and benign breast diseases such as mastitis and fibroadenoma of the breast are more common causes of breast disorder symptoms [78].Nevertheless, the appearance of a new symptom should be taken seriously by both patients and their doctors, because of the possibility of an underlying breast cancer at almost any age[79].

\section{Detection and Diagnostic Markers}

Two most commonly used screening methods, physical examination of the breasts by the healthcare professional and mammography, can offer an approximately likelihood that a lump is a cancer, and may also detect some other lesions, such as a simple cyst [80]. When the examinations are inconclusive, a fine needle aspiration or fine needle aspiration and cytology-FNAC, is performed to establish the diagnosis. A finding of clear fluid makes the lump highly unlikely to be cancerous, but bloody fluid may be sent for microscopy examination for cancerous cells (cytological examination). Together with physical examination of the breasts, mammography, and FNAC can be used to diagnose breast cancer with a good degree of accuracy. Other options for biopsy include a core biopsy or vacuum-assisted breast biopsy [81].

\section{Breast cancer Classification}

Various grading systems are used to classify breast cancers:

Histopathological examination.Breast cancer is usually classified primarily by its histologicalappearance. Most breast cancers are derived from the epithelial lining the ducts or lobules, and these are classified as ductal or lobular carcinoma. Carcinoma in situ is growth of low grade cancerous or precancerous cells within a 
particular tissue compartment such as mammary duct without invasion of surrounding tissue.Incontrast, invasive carcinoma does not confine itself to the initial tissue compartment [82].

Grading breast cancer cells.Grading compares the appearance of the breast cancer cells to the appearance of normal breast tissue. Normal cells in an organ like the breast become differentiated, meaning that they take on specific shapes and forms that reflect their function as part of that organ. Cancerous cells lose that differentiation. In cancer, the cells that would normally line up in an orderly way to make up the milk ducts become disorganized. Cell division becomes uncontrolled. Pathologistsdescribe cells as well differentiated (low grade), moderatelydifferentiated (intermediate grade), and poorly differentiated(high grade) as the cells progressively lose the features seen in the normal breast cells[83,84].

\section{Staging breast cancer}

TNM system is used in breast cancer staging,T-tumor,N,lymphnodes, and M tumor metastasized(cancer spread to other body parts).Frequently used staging are:

a). Zero stage, is a pre-cancerous or marker condition, either ductal carcinoma in situ(DCIS) or lobular carcinoma in situ(LCIS)

b).Stage 1-3 is within the breast or regional nodes

c).Stage 4 is 'metastatic 'cancer that has less favorable prognosis since it has escaped into the bloodstream. In breast cancer patient with low risk for metastasis, the risks associated with PET(position emission tomography),CT scan, or bone scans outweigh the possible benefits, as these procedures expose the patient to substantial amount of potentially ionizing radiations[83,84].

Receptor status; Breast cancer cells may or may not have three important receptors: estrogen receptor(ER), progesteronereceptor(PR), and HER2[85].DNA testing of various types including DNA microarrays have compared normal cells to breast cancer cells[86].

\section{Therapy}

Therapy or management of breast cancer depends on various factors, including the stage of the cancer and the age of the patient. Breast cancer is usually treated with surgery, which may be followed by chemotherapy or radiation therapy or both. A multidisciplinary approach is preferable [87].Hormone receptorpositive cancers are often treated with hormone-blocking therapy over courses of several years. Monoclonalantibodies, or other immune-modulating treatments, may be administered in certain cases of metastatic and other advanced stages of breast cancer [87].Frequently surgeries include: Mastectomy: Removal of whole breast.Quadrantectomy:Removal of one quarter of the breast. Lumpectomy: Removal of a small part of the breast [87].

\section{Prognosis and Prevention}

Prognosis is usually given for the probability of progression-free survival (PFS) or disease free survival(DFS).Survival is usually calculated as an average number of months(years) that $50 \%$ of patients survive, or the percentage of patients that are alive after,1,5,15, and 20 years. Prognosis is important for treatment decision because patients with a good prognosis are usually offered less invasive treatments, such as lumpectomy and radiation or hormone therapy, while patients with poor prognosis are usually offered more aggressive treatment, such as more extensive mastectomy and more chemo therapy drugs. The Nottingham Prognostic Index is a commonly used prognostic tool [88].Breast cancer staging takes into consideration size, localinvolvement, lymph node status and whether metastatic disease is present. The higher the stage at diagnosis, the poorer the prognosis[88].Younger women with an age of less than 40 years or women over 80 years tend to have a poorer prognosis than post-menopausal women due to several factors[89].

\section{Prevention}

Change in life style include maintaining health weight, consuming less alcohol, physically active and breast feeding their children[13]. These modifications might prevent $38 \%$ of breast cancers in US, $42 \%$ in the UK, $28 \%$ in Brazil and $20 \%$ in China[89].Intake of marine omega -3 polyunsaturated fatty acids and consumption of soy-based foods may reduce risk[90,91].Prophylactic mastectomy(removal of both breasts) may be considered in people with BRCA1 and BRAC2 mutations, which are associated with a substantially heightened risk of for an eventual diagnosis of breasts cancer[92]. The selective estrogen modulators(such as tamoxifen) reduce the risk of breast cancer but increase the risk of thromboembolism and endometrial cancer[93]. Breast cancer Preventive task force recommends mammography between the ages50 and 74 year [94].The council of Europe recommends mammography between 50 and 69 year, with a 2- year frequency. In Canada screening recommended between ages of 50 and 74 at a frequency of 2 to 3 years[95,96].The task force reports point out that in addition to unnecessary surgery and anxiety, the risks of more frequent mammograms include a small but significant increase in breast cancer induced radiation [97]. 


\section{Conclusion}

Breast cancer is the most common cause of cancer in women with high mortality worldwide. Incidence of breast cancer is high in Europe and in North America and lowest in Africa and Asia. Risk factors for developing breast cancer include obesity, lack of exercise, alcohol consumption, oral contraceptive, hormone replacement therapy, and family history. Therapy mainly by surgery, medication, and radiation.Early diagnosis has better outcome. Prevention by life style changes.

[1]. Breast Cancer.NCI.Retrieved 29 June 2014.

\section{References}

[2]. Breast Cancer Treatment (PDQ®).NCI.23 May 2014.Retrieved 29 June 2014.

[3]. MacGuireA,BrownJA,MaloneC,etal.Effects of age on the detection of breast cancer.Cancers.2015;7(2):908-29.

[4]. World Cancer Report.International Agency for Research on Cancer.2008.Retrieved 26 February 2011.

[5]. World Cancer Report 2014.International Agency for Research on Cancer. World Health Organization.2014.ISBN978-92-8320432-9.

[6]. World Cancer Report 2014.Worlf Health Organization.2014.pp.Chapter 1.1.ISBN 92-832-0429-8.

[7]. Stewart BW,Kleijues P.(Eds):World Cancer Report.IARCPress.Lyon 2003 (http:// www.scribd.com/doc/2350813/world-CancerReport-2003-Stuart-e-Kleihues-WHO-e-IARC)

[8]. Zainal A0,ZainudinMA,NorSaleha IT. Malaysian Cancer Statistics-Data and Figure Peninsular Malaysia. National Cancer Registry, Ministry of Health,2006:8.

[9]. Breast Ca:Breast Cancer in Young Women(http://www.webmd.com/breast-cancer/ guide/breast-cancer young women-)WebMDRetrieved 9 September 2009.

[10]. Breast Cancer Treatment(PDQR).NCI.26 June 2014.Retrieved 29 June 2014.

[11]. World Cancer Report (PDF).International Agency for Research on Cancer. 2008.Retrieved 26 February 2011.

[12]. Cancer survival in England:Patients Diagnosed 2007-2011 and followed up to 2012(PDF).Office for National Statistics.29 October 2013.Retrieved 29 June 2014.

[13]. American Institute for Cancer Research/World Cancer Research Fund, Food, Nutrition, Physical Activity and the Prevention of Cancer: a Global Perspective)http://www.dietandcancerreport.org).

[14]. Oldest evidence of breast cancer found in Egyptian Skeleton.Reuters.24 March 2015.Retrived 25 March 2015.

[15]. History of Cancer.American Cancer Society.25 March 2002.Retrieved 9 0ctober 2006.

[16]. Olson 2002,pp.32-33.

[17]. YalomMarilyn(1997).A history of the breast..NewYork:Alfred A.Knopf.p.234.ISBN 0-679-43459-3.

[18]. Aronowitz RA.(2007).Unnatural history:breast cancer and American Society.Cambridge,UK:Cambridge University Press.pp.2224.

[19]. Olson JS(2002).Bathsheba's breast:women,cancer\&history.Baltimore:The John Hopkins University Press.pp.9-13.ISBN 0-80186936-6.

[20]. History of Breast Cancer.Random History.27 February 2008.Retrieved 8 May 2010

[21]. Olson 2002.pp.102-6.

[22]. Olson 2002,p.1.

[23]. Marc Lacroix(2011).A Concise History of Breast Cancer.USA:Nova Science Publication.pp.59-68.ISBN 978-1-1-61122-305-7.

[24]. Alfredo Morbia(2004).A History of Epidemiologic Methods and Concepts. Boston:Birkhauser.pp.301-302.ISBN 3-7643-68187.Retrieved 31 December 2007.

[25]. Sulik GA.(2010).Pink Ribbon Blues:How Breast Cancer Culture UnderminesWomen'sHealth.USA:Oxford University Press.pp.200-3.ISBN 0-19-974045-3.OCLC 535493589.

[26]. Hayes J,RichardsonAnn,FramptonC.Population attributable risks for modifiable lifestyle factors and breast cancer in New Zealand women.JMJ.2013;43(11):1198-1204.

[27]. Reeder JG,VogelVG.Breast Cancer prevention.Cancertreatment and research. 2008;141:149-64.

[28]. Am I at risk.Breast Cancer Care.Rerieved 22 October 2013.

[29]. Collaborative Group on Hormonal Factors in Breast Cancer.Breast cancer and breastfeeding:Collaborative reanalysis of individuals data from 47 epidemiological studies in 30 countries,including 50302 women with breast cancer and 96973 women without the disease.Lancet.2002;360(9328):187-95.

[30]. YagerJD,DavidsonNE.Estrogen carcinogenesis in breast cancer.NewEngl JMed.2006;354(3):270-82.

[31]. MazzuccoA,SantoroE,DeSotoM,et al.(2009).Hormone Therapy and Menopause.National Research Center for Women \& families.

[32]. Light Pollution as new risk factor for human Breast and ProstateCancers-Haim,Abraham;Portnov,Bins P.2013,ISBN 978-94-0076220-6.

[33]. JohsonKC,MillarJ,TurcotteF.Active smoking and second smoke increase breast cancer risk: the report of Canadian Expert Panel on Tobacco Smoke and Breast Cancer Risk.Tobacco control.2011;20(1):e2.

[34]. Lee IM,ShiromaEJ,LobeloF,etal.Effect of physical activity on major non-communicable diseases worldwide:an analysis of burden of disease and life expectancy.The Lancet.2012;380(9838):219-29.

[35]. Biswas A,OhPI,FaulknerGE,etal.Sedendary Time and Its association With Risk for Disease Incidence,Mortality and Hospitalization in Adults: A Systematic Review and Meta -Analysis.Ann Internal Med.2015;162(2):123-32.

[36]. VeljkovicM,VeljkovicS.The risk of breast cervical,endometrial and ovarian cancer in oral contraceptive users.Medicinskipregled.2010;63(9-10):657-61.

[37]. Casey PM,CerhanJR,PruthiS.Oral contraceptive use and risk of breast cancer. Mayo Clinic proceedings.MayoClinic;2008;83(1):86-90;quiz 90-1.

[38]. IodiceS,BarileM,RotmenszN, etal.Oral contraceptive use and breast ovarian cancer risk in BRCA1/1 carriers: a metaanalysis.European journal of cancer(OxfordEngland:1990).2010;46(12):2275-84.

[39]. Yang I.Jacobson KH.A systematic review of the association between breastfeeding and breast cancer. $J$ Women'sHealth.2008;17(10):1635-45.

[40]. Russo J,RussoIH.Susceptibility of the mammary gland to carcinogenesis. 11.Pregnancy interruption as a risk factor in tumor incidence.Am JPathol.1980;100(2):505-06. 
[41]. BeralV,BullD,DollR,etal.Breast cancer and abortion:collaborative reanalysis of data from 53 epidemiological studies,including 83,000 women with breast cancer from 16 countries.Lancet.2004;363(9414):1007-16.

[42]. Blackburn GI,WangKA.Dietary fat reduction and breast cancer outcome:results from the women's Nutrition Intervention Study(WINS).Am J Clin Nutrition. 2007; 86(3):s878-81.PMID 18265482.

[43]. Boffettap,HashibeM,LaVecchiaC,etal.The burden of cancer attributable to alcohol drinking Int J Cancer.2006;119(4):884-7.

[44]. BBC report Weight link to breast cancer (http:// ncws .bbc. co.uk///hi/health/517838.stm).

[45]. ScceveC,AnguianoB,Delgado G.Is iodine a gatekeeper of the integrity of the mammary gland.J Mammary Gland Biology andNeoplasia.2005;10(2):189-96.

[46]. MouroutiN,KontogianniMD,PapavagelisC,etal.Diet and Breast cancer:a systematic review.Int $J$ Food SciandNutrition.2015;66(1):1-42.

[47]. AuberyAllison.A Diet High In Fiber May Help Protect Against Breast Cancer.NPR.Retrieved 1 February 2016.

[48]. Boris Pasche.Cancer Genetics (Cancer Treatment and Research). Berlin: Spinger. pp.19-20.ISBN 1-4419-6032-5.

[49]. Gage M,WattendorfD,HenryLR.Translational advances regarding hereditary breast cancer syndrome.JSurg onocol.2012;105(5):444-51.

[50]. Colditz Graham A,KaphinstKA,HankisonSE,etal.Family history and risk of breast cancer:nurse's health study.Breast CancerResearch Treatment.2013;133(3):1097-1104.

[51]. Familial breast cancer:collaborative reanalysis of individuals data from 52 epidemiological studies including 58,209 women with breast cancer and101,986 women without the disease.Lancet.2001;358(9291):1389-99.

[52]. Nelson HD,ZakherB,CantorA,etal.Risk factors for breast cancer for women aged 40 to 49 years: a systematic review and metaanalysis. Annals of Int Med.2012;156(9):635-48.

[53]. KolataGina.Genetic Study Finds 4 Distinct Variations of Breast Cancer.The New York Times.Retrieved September 2012.

[54]. American Cancer Society(2005).Breast Cancer Facts \& Figures 2005-2006(PDF) Archived from original(PDF) on 13 June. Retrieved 26 April 2007.

[55]. Wand XS,ArmstrongME,CaimsBJ,etal.Shift work and chronic disease: the epidemiological evidence. Occup Med.2011;61(2):7889.

[56]. Brody JG,RudelRA,MichelKB,etal.Environmentalpollutants,diet,physicalactivity,bodysize,and breast cancer:where do we stand in research to identify opportunities for prevention.Cancer.2007;109(12 Suppl):2627-34.

[57]. HendrickRE.Radiation doses and cancer risks from breast imaging studies.Radiology.2010;257(1):246-53

[58]. Understanding Breast Changes.National Cancer Institute.archieved from the original on 27 May 2010.

[59]. Lobular carcinoma in situ.Marker for breast cancer risk.Mayo Clinic.com.

[60]. AnothaisintaweeT,WirakampunC,LerdsitthichaiP, etal.Risk factors of breast cancer:a systematic review and metaanalysis.AsianPcafic J PubHealth.2013;25(5):368-87.

[61]. Adrian Lee,CarlosArtega (14 December 2009).32 ${ }^{\text {nd }}$ Annual CTRC-AACR San Antonio Breast Cancer Symposium(PDF).SundayMorning year End Review. Achieved from the original (PDF)on 13 August 2013.

[62]. CavalieriE,ChakravartiD,GuttenplanJ,etal.Catechol estrogen quinones as initiators of breast and other hunancancers:implications of biomarkers of susceptibility and cancer prevention.Biochimica et Biophysica Acta.2006;766(1):63-78.

[63]. Haslam SZ,WoodwardTL.Host microenvironment in breast cancer developmeny epithelial cell-stromal-cell interactions and steroid hormone in normal and cancerous mammary gland.Breast Cancer Res.2003;5(4):208-15.

[64]. Jarde T, PrerrierS,VassonMP, etal.Molecular mechanism of leptin and adiponectin in breast cancer.Eur J Cancer.2011;47(1):3343.

[65]. BeggCB,HaileRW,BorgA, et al. Variation of breast cancer risk among BRCA1/2 carriers.JAMA.2008;299(2):194-201.

[66]. Patel KJ,YUVP,LeeH, etal.INvolevement of brca2 in DNA repair.Mol Cell.1998;1(3):347-57.

[67]. Marietta C,ThompsonLH,LamerdinJE,etal.Acetaldehyde stimulates FANCD2monoubiquitination H2AX phosphorylation and BRCA 1 phosphorylation in human cells in vitro: implications for alcohol-related carcinogenesis. Mutat Res. 2009; 644(1-2):7783 .

[68]. Wooster R,WeberBL.Breast and ovarian cancer.NEngl J Med.2003;348(23):2339-47.

[69]. Levin B,Lech D, Friedensen B. Evidence that BRCA 1-or BRCA2-associated cancers are not inevitablw.Mol Med.2012;18:132737.

[70]. KourosMH,KimJW,BechisSK, et al.GATA-3 and the regulation of the mammary luminal cell fate.CurrOpin cellbiology.2008;20(2):164-70.

[71]. Merck Manual of Diagnosis and Therapy(February 2003).Breast Disorders:BreastCancer.Retrieved 5 February 2008.

[72]. American Cancer Society(2007).Cancer Facts \& figures 2007(PDF).Archieved from the original (PDF) on April 2007.Retrieved 26 April 2007.

[73]. Watson M.Assessment of suspected cancer.InnoAiT.2008;1(2):94-107.

[74]. eMedicine(23 August 2006)Breast Cancer Education.Retrieved 5 February 2008.

[75]. National Cancer Institute (27 June 2005).Paget's Disease of the Nipple: Questions and Answers. Retrieved 6 February 2008

[76]. Lacroix M.Significance detection and markers of disseminated breast cancer cells.Endocrine RelatedCancer(Bioscientifica).2006;13(4):1033-67.

[77]. National Cancer Institute(1 September 2004).Metastatic Cancer.Questions and Answers .Retrieved 6 February 2008.

[78]. Interpreting Signs and Symptoms.Lippincott Williams \& Wikins.2007.pp.99-ISBN 978-1-58255-668-0.

[79]. Merk Manual of Diagnosis and Therapy(February 2003).Breast Disorders:Overview of BreastDisorders.Retrieved 5 February 2008.

[80]. SaslowD,Hannan J,0sush J,etal.Clinical breast examination:practical recommendations for optimizing performance and reporting.CA:A Cancer J Clinicians. 2004; 54(6):327-44.

[81]. YU YH,LiangC,YuanXZ.Diagnostic vale to vacuum-assisted breast biopsy for breast carcinoma: a meta-analysis and systematic review. Breast Cancer reseach andtreatment.2010;120(2):469-79.

[82]. Merck Manual.ProfessionalEdition(http://www.merck manuals. com/professional/ gynecology_and_obstetrics/breast_disorders/breast_cancer.html).Ch.253,Breast Cancer.

[83]. American Society of Clinical Oncology,Five Things Physicians and Patients Should Question. Choosing Wisely an initiative of ABIM Foundation (American Society of Clinical Oncology),retrieved 14 August 2012.

[84]. Carlson RW,AllredDC,AndersonBO,etal.Breastcancer.Clinical practice Guidelines in oncology.J Nat Comprehensive CancerNetwork:JNCCN.2009;7(2):122-192.PMID 19200416.

[85]. Kumar Vinay,AbdulAbas.Robins and Cotran Pathologic Basis of Disease. Philadelphia:Saunders,an imprint of Elsevier inc.p.1090.ISBN 978-1-4160-3121-5. 
[86]. RomondEH,PerezEA,BryantJ,etal.Trastuzummab plus adjuvant chemptherapy for operable HER2-positive breast cancer.NEngl JMed.2005;353(16):1673-84.

[87]. Saini KS,TaylorC,RamirezAJ,etal.Role of multidisciplinary team in breast cancer management: results from a large international survey involving 39 countries.Annalsof Oncology.2013;23(4):853-9.

[88]. Breast Cancer:BreastDisorders:Merck Manual Professional.Merck.com.Retrieved 8 Mat 2010.

[89]. PeppercomJ.Breast Cancer in Women Under 40.Oncology.2009;23(6).

[90]. AhengJS,HuZJ,Zhao YM.et al.Intake of fish and marine n-3 polyunsaturated fatty acids and risk of breast cancer:meta- analysis of data from 21 independent prospective coghort studies.BMJ.2013;346:f3706.

[91]. Wu AH,YuMC,YsengCC,etal.Epidmiology of soy exposures and breast cancer risk.Brit J Cancer.2008;98(1):9-14 .

[92]. Hartmann LC,SchaidDJ,WoodsJE,etal.Efficacy of bilateral mastectomy in woman with a family history of breast cancer.NEnglJMed.1999;340(2):77-84.

[93]. Nelson HD,SmithME,GriffinJC, etal.Use of medications to reduce risk for primary breast cancer:a systematic review for the U.S. Preventive Services Task Force.Annals of Internal Med.2013;158(8):604-14.

[94]. Siu Albert L.Screening for Breast Cancer:U.S.Preventive Services Task Force Recommendations Statement. Annals of InternalMed.201.Doi:10.7326/M15-2886.

[95]. BiesheuvelC,WeigelS,HeindelW.MammographyScreening:Evidence,History and Current Practice in Germany and Other European Countries.. Breast Care(BaselSwitzerland)2011;6(2):104-109.

[96]. Tonelli M,ConnorGS,JoffresM,etal.Recommendations for screening for breast cancer in verage risk women aged $40-74$ years.Canadian Med Assoc J.2011;183(17):1991-2001.

[97]. Breast Cancer" Screening. United States Preventive Services Task Force.Archieved from the original on 16 June 2013. 